\title{
ON THE INTEGRO-DIFFERENTIAL EQUATION OF THE BÔCHER TYPE IN THREE-SPACE
}

\author{
BY G. E. RAYNOR
}

1. Introduction. Bôcher has shown* that if a function $f(x, y)$ is continuous and has continuous first partial derivatives in a region $R$ and satisfies the condition

$$
\int_{C} \frac{\partial f}{\partial n} d s=0
$$

for every circle $C$ lying entirely in $R$, then $f(x, y)$ is harmonic at each interior point of $R$. Bôcher treats only functions in two variables and by a method which cannot be directly extended to three-space.

It is the purpose of the present note to show, by a simple modification of the second part of Bôcher's argument, that this result may at once be extended to three-space, and also to investigate the nature of the function $f$ if Bôcher's condition of continuity is somewhat weakened. We shall treat explicitly functions in three variables only, but it will easily be seen that with a slight modification the statements of Theorem II are applicable to two-space as well.

THEOREM I. If a function $f(x, y, z)$ is continuous, and has continuous first partial derivatives in a connected finite region $R$, and is such that the surface integral $\int_{S}(\partial f / \partial n) d s$ vanishes when taken over every sphere $S$ lying in $R$, then at each interior point of $R, f$ is harmonic; that is, it satisfies Laplace's equation

$$
\frac{\partial^{2} f}{\partial x^{2}}+\frac{\partial^{2} f}{\partial y^{2}}+\frac{\partial^{2} f}{\partial z^{2}}=0
$$

at each interior point of $R$.

- Proceedings of the American Academy, vol. 41, pp. 577-583. 
In the preceding integral, as well as in what follows, the derivative $\partial f / \partial n$ is to be taken either toward the interior of $S$, or toward the exterior of $S$, throughout the region of integration. Let $P$ be any interior point of $R$ and consider two spheres $S_{1}$ and $S_{2}$ of radii $r_{1}$ and $r_{2}<r_{1}$ with centers at $P$. By hypothesis, we have

$$
\int_{S} \frac{\partial f}{\partial n} d s=0
$$

or, setting $d s=r^{2} d \omega$, where $d \omega$ is the element of area on the unit sphere with center $P$,

$$
\int_{S} \frac{\partial f}{\partial n} d \omega=0
$$

It follows that

$$
\int_{r_{2}}^{r_{1}} d r \int_{S} \frac{\partial f}{\partial n} d \omega=0
$$

Because of the continuity of $f$ and its derivatives the order of integrations in the above integral may be inverted and we have

$$
\int_{S_{1}} f d \omega-\int_{S_{2}} f d \omega=0
$$

Let $f(P)$ be the value of $f$ at the point $P$. Then since $f$ is continuous at $P$ we obtain from (2) by letting $r_{2}$ approach zero,

$$
f(P)=\frac{1}{4 \pi} \int_{s_{1}} f d \omega=\frac{1}{4 \pi r_{1}^{2}} \int_{S_{1}} f d s .
$$

We thus see that our function $f$ possesses the so-called meanvalue property, that is, its value at the center of any sphere is the mean of its values on the surface of the sphere.

Consider now the function $F$ which takes the same values as $f$ on $S_{1}$ and which is harmonic interior to $S_{1}$. This function exists and can be expressed as a Poisson integral. It is well known that $F$ also possesses the mean-value prop- 
erty and hence so also does the difference $f-F$. But a continuous function having the mean value property in a closed region $R$ must take its greatest and least values on the boundary of $R$. Since the difference $f-F$ is identically zero on $S_{1}$ it follows that it is zero everywhere within $S_{1}$ and hence $f$ must be harmonic at $P$ as was to be proved.

It is evident that the original hypothesis that

$$
\int \frac{\partial f}{\partial n} d s=0
$$

about every sphere in $R$ is unnecessarily broad. All that is needed in the above proof is that each point $P$ may be surrounded by a region, no matter how small, which is such that the above integral vanishes when taken over every sphere lying entirely within it.

2. A More General Theorem. We shall now weaken the original condition of continuity on $f$ and suppose that it is continuous at every interior point of $R$ except possibly at a finite number of points $P_{1}, P_{2}, \cdots, P_{i}, \cdots, P_{n}$. We shall refer to these exceptional points in the sequel as the points $P_{i}$. Our other condition on $f$ now takes the form "about each interior point of $R$ there exists a region $M$ which is such that in its interior $\int(\partial f / \partial n) d s$ evaluated over every sphere which lies in $M$ and does not pass through one of the $P_{i}$ is zero." It is sufficient that if $M$ contains one of the exceptional points, it contains only one.

That $f$ is harmonic at every interior point $P$ of $R$ other than the $P_{i}$ follows readily. About each of the $P_{i}$ as center draw a small sphere $S_{i}$ which does not contain $P$. Then the region bounded by the $S_{i}$ and the boundary of $R$ is a region of the type considered in Theorem I from which it follows that $f$ is harmonic at $P$. It thus remains only to consider the nature of $f$ in the neighborhood of any one of the $P_{i}$.

In a paper presented to the Society, October 31, 1925, the writer has shown that if a function is harmonic at every point 
in the deleted neighborhood of a point $P$ it may be expressed in the form

$$
c \frac{1}{r}+\Phi(x, y, z)+V(x, y, z)
$$

in this neighborhood. In this expression $c$ is a constant, $r$ the distance from $P$ to $(x, y, z), V$ a function harmonic everywhere in the neighborhood of $P$ as well as at $P$ itself and $\Phi$ a function harmonic in the deleted neighborhood and such that it is either identically zero or else there exist modes of approach to $P$ for which $\Phi$ will tend toward plus infinity and also modes of approach for which it will tend toward minus infinity; $\Phi$ also possesses the property that its integral over the surface of any sphere with $P$ as center vanishes.

Consider now two spheres $S_{1}$ and $S_{2}$ with center $P$ and radii $r_{1}$ and $r_{2}<r_{1}$. Apply Green's formula to the functions $\Phi$ and $1 / r-1 / r_{1}$ for the region bounded by $S_{1}$ and $S_{2}$ and we have

$$
\int_{S_{1} S_{2}}\left\{\left(\frac{1}{r}-\frac{1}{r_{1}}\right) \frac{\partial \Phi}{\partial n}-\frac{\partial}{\partial n}\left(\frac{1}{r}-\frac{1}{r_{1}}\right) \Phi\right\} d s=0,
$$

where the normal derivatives are taken toward the interior of the region $S_{1} S_{2}$. Remembering that the integral of $\Phi$ over any sphere with center $P$ is zero and since $1 / r-1 / r_{1}$ is zero on $S_{1}$ and constant on $S_{2}$ we have from the above equation

$$
\int_{S_{2}} \frac{\partial \Phi}{\partial n} d s=0
$$

Since $S_{2}$ is any sphere interior to $S_{1}$ it follows that the integral of the normal derivative of $\Phi$ over any sphere with center $P$ and radius less than $r_{1}$ vanishes. The same result could of course be obtained from the property $\int_{S} \Phi d s=0$ by considering the continuity of $\Phi$ and using the theorem concerning the differentiation of a definite integral. In (3) because of the continuity of the first partial derivatives of $\Phi$ in the deleted neighborhood of $P$ we may take the normal derivative either toward the interior or toward the 
exterior normal of $S_{2}$ throughout the region of integration. Hence if the function

$$
f=c \frac{1}{r}+\Phi+V
$$

is to be such that the integral of its normal derivative vanishes when taken over spheres in the neighborhood of $P$, the constant $c$ must be zero. Conversely we have shown by the above argument that if $c$ is zero $\int(\partial f / \partial n) d s$ will vanish when taken over every sphere with center $P$ and of radius $r<r_{1}$. We may now state the following theorem.

THEOREM II. Every function which satisfies the conditions of $\S 2$ in a region $R$ is harmonic at every interior point of $R$ except possibly at the points $P_{i}$. In the neighborhood of each $P_{i}, f$ is of the form $\Phi+V$. If $\Phi \equiv 0$, in the neighborhood of any $P_{i}, P_{i}$ is at most a removable discontinuity. If $\Phi \neq 0$, $f$ will be harmonic in the deleted neighborhood of $P$ and will be such that for certain modes of approach to $P$ it will tend toward plus infinity and for other modes to minus infinity.

It may be remarked in closing that although we have supposed $\int(\partial f / \partial n) d s$ to vanish only when taken over sufficiently small spheres with $P_{i}$ as center it is now easy to prove that it will vanish when taken over any regular surface $S$ in $R$ which does not pass through one of the $P_{i}$. We need merely to surround each $P_{i}$ in $S$ by a sphere $S_{i}$ lying entirely in $S$ and use the fact that

$$
\int \frac{\partial f}{\partial n} d s=0
$$

where the integral is taken over $S$ and the spheres $S_{i}$. Then $\int_{S}(\partial f / \partial n) d s$ will vanish since the portion of (4) due to the $S_{i}$ vanishes.

WESLEYAN UNIVERSTTY 